\title{
On Strength of Brittle Nanomaterials: Confinement Effect on Weibull Distributions
}

\author{
Dahye Shin and Dongchan Jang*
}

Department of Nuclear and Quantum Engineering Korea Advanced Institute of Science and Technology (KAIST), Daejeon, South Korea

Recent progress in nanotechnology enables us to utilize elastic strain engineering, the emerging technology capable of controlling the physio-chemical properties of materials via externally-imposed elastic strains, for hard materials. Because the range of properties accessible with elastic strains are set by materials' elasticity limits, it is of great importance to suppress the occurrence of any inelastic deformations and failure, and thus the fundamental knowledge on fracture behavior at nanoscale is highly required. The conventional Weibull theory, which has been widely used for last the few decades to explain the failure statistics of brittle bulk materials, has a limitation to be directly applied to samples of nanometer dimensions because the baseline assumption on statistical equivalence becomes intractable for small samples. In this study, we suggest

OPEN ACCESS

Edited by:

Federico Bosia,

University of Turin, Italy

Reviewed by:

Paolo S. Valvo,

University of Pisa, Italy

Giuseppe Puglisi,

Politecnico di Bari, Italy

${ }^{*}$ Correspondence:

Dongchan Jang

dongchan.jang@kaist.ac.kr

Specialty section:

This article was submitted to

Mechanics of Materials,

a section of the journal

Frontiers in Materials

Received: 01 August 2019

Accepted: 30 October 2019

Published: 15 November 2019

Citation:

Shin D and Jang D (2019) On Strength of Brittle Nanomaterials:

Confinement Effect on Weibull Distributions. Front. Mater. 6:289.

doi: 10.3389/fmats.2019.00289 an integrated equation presenting the sample size effect on fracture strength for brittle nanomaterials by further considering the confinement of the flaw size distribution. This new approach is applicable to any homogeneous brittle nanomaterial whose failure is governed by linear elastic fracture mechanics and shows good agreement with experimental data collected from the literature. We expect that this theoretical study offers a new guideline to employ brittle nanomaterials in designing and fabricating the advanced strain engineering system.

Keywords: brittle, confinement effect, fracture strength, nanoscale fracture, nanoscale Weibull distribution, size effect

\section{INTRODUCTION}

Thermodynamic potentials and free energies of elastically-deformed solid bodies explicitly depend on the strains or stresses (Kittel and McEuen, 1996; Gilman, 2003). This fact, in principle, offers a unique opportunity for materials engineers to tune and optimize many physical and chemical properties of functional materials by externally imposing elastic strains (Zhu and $\mathrm{Li}, 2010 ; \mathrm{Li}$ et al., 2014). One necessary condition to take the utmost benefit from this strategy is large elastic deformability while suppressing failure by inelastic deformation or fracture so as to maximize the accessible domains in materials' design parameter space. This condition is only marginally fulfilled for conventional hard materials, for their strain values at yield or fracture are typically $<1 \%$ (Pelleg, 2014; Anderson, 2017). On the other hand, recent studies on nanomechanics revealed that both yield (Jang and Greer, 2010; Wang et al., 2013) and fracture (Suresh and Li, 2008; Jang et al., 2013) strengths of many hard nanomaterials drastically increase, up to a significant fraction of their ideal strengths, and accordingly the elasticity limits increase as well, when the sample sizes decrease down 
to below a few 100 nanometers. This strengthening effect is the purely size-induced one without involving any microstructural modification and therefore gives us the additional possibility to broadly adopt the method of strain engineering as long as we can manage to keep their dimensions at the nanoscale.

In most practical cases, fabrication of a specimen entirely free from defects and flaws is almost unachievable, and its strength and elastic limit decrease substantially due to those imperfections (Anderson, 2017). In this regard, it is of great importance to properly understand and reliably predict the occurrence of an inelastic failure in the presence of pre-existing flaws in order to fully utilize elastic strain engineering for hard materials. In general, under the scheme of linear elastic fracture mechanics (LEFM), Weibull analysis based on the weakest link theory welldescribes the strength and failure of brittle materials (Weibull, 1951; Quinn and Quinn, 2010). In this approach, the fracture strength depends on the sample volume following the inverse power-law relationship whose exponent is usually called Weibull modulus (Quinn and Quinn, 2010). Its linkage to the weakest link theory is easily understandable once we consider the fact that larger specimens likely contain more imperfections than smaller ones and hence have a higher probability of including more fatal flaws, which is the largest according to LEFM (Anderson, 2017). However, without restricting the upper bound for the flaw sizes to be, this theory, in its original form, has some limitations to be directly used for tiny samples in which the method of strain engineering is likely to work most efficiently. Conventional Weibull theory assumes sufficiently small flaws in comparison with the size of specimen to ensure the statistical equivalence of a randomly-chosen sub-sample, i.e., an arbitrarily small volume defined as a part of the entire sample, but this condition becomes intractable for nanomaterials as their external dimensions decrease approaching the typical flaw sizes existing in the material. Alternatively, there have been several attempts to establish theoretical foundations for the statistical determination of fracture strength of nanomaterials. For example, taking the effects from the temperature and strain rate into account, Sun et al. and Taloni et al. modified the conventional Weibull model to incorporate with plasticity in some nanomaterials (Sun et al., 2012; Taloni et al., 2018). Pugno and Ruoff recognized the discreteness of flaw characteristics at the atomic scale and developed a theory explaining the size-independent fracture strength in nearly defect-free nanomaterials containing only a few atomic vacancies (Pugno and Ruoff, 2006). Treating the contributions from the surface (2D) and the interior (3D) of the samples separately, several researchers regarded some nanomaterials as effectively having the non-integer dimensions and proposed the fractal dimensional dependency (Carpinteri and Pugno, 2004, 2005; Pugno and Ruoff, 2006) for the quantized crack propagation (Pugno and Ruoff, 2004) and fractal size effects (Carpinteri, 1994). On the other hand, there are other studies focused on the quasi-brittle nature of nano-sized samples because their comparable fracture process zone size becomes non-negligible even for macroscopically brittle (Pang et al., 2008; Le and BaŽant, 2012). However, none of the aforementioned theories have integrated the variation of the fundamental statistical characteristics affected by the volumetric confinement

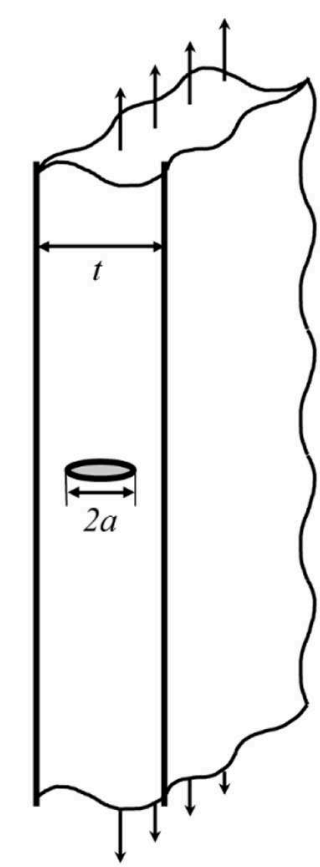

FIGURE 1 | Schematic illustration of a cracked plate under uniaxial tensile load.

in the tiny nano-scaled specimens. In this study, we derived an integrated equation describing the influence of size on the fracture strength of brittle nanomaterials, based on conventional Weibull statistics but further considering confinement effects on the flaw size distribution set up by the external dimensions. We confirmed the validity of this approach by comparing our predictions with the experimental data collected from the literature (Hoffmann et al., 2007; Richter et al., 2009; Xu et al., 2010; He and Zhu, 2011). We expect that this theoretical study offers a new guideline to design the enhanced strain engineered system composed of brittle nanomaterials.

\section{EXTREME VALUE DISTRIBUTIONS FOR FLAW SIZES AND FRACTURE STRENGTHS OF BRITTLE MATERIALS}

According to LEFM, the fracture strength of brittle materials $\sigma_{\mathrm{f}}$, inversely scales with the square root of the existing flaw size $a$ (Anderson, 2017):

$$
\sigma_{f}=\frac{K_{I C}}{\sqrt{\pi a}} F(\varphi)
$$

where $K_{\mathrm{IC}}$ is the fracture toughness, and $F(\phi)$ is a correction factor taking finite sample size effects into account and given as a function of relative flaw size with respect to the sample dimension ( $\phi=a / t$, see the schematics in Figure 1). This LEFM formalism clearly indicates the explicit dependence of the fracture strength on the flaw size for a given $K_{\mathrm{IC}}$, following an inverse square-root 
relationship, i.e., the larger the flaw is, the weaker the material becomes. In reality, a number of flaws with different sizes exist within the material, out of which the largest one determines the actual strength of the sample in a constant stress field because fracture initiates there at the lowest far-field load. In this sense, the strength of a brittle material is not an intrinsic property but rather stochastically determined by the statistical nature of flaw sizes. Because it is the largest flaw that matters for the fracture strength, but the smaller ones are of little interest, a statistical model expressed in terms of extreme values (Coles et al., 2001; Quinn and Quinn, 2010) is required.

One of such formalism, called Weibull statistics, is wellrecognized as an appropriate model for the fracture strength of brittle materials due to its functional simplicity, the physical requirement to be able to predict zero strength, and most importantly excellent agreement with experimental data (Quinn and Quinn, 2010). In his seminal work (Weibull, 1951), Weibull established the extreme value formulation of fracture strength, in which the statistical random variable is parameterized by flaw strength $\sigma_{a}$, the conceptual value associated with individual flaw by Equation (1). Then, considering a brittle solid as being divided into many statistically-equivalent sub-samples of uniform volume $V_{0}$ (see Figure $2 \mathrm{~A}$ ), the probability $P_{V 0}$, for this volume element containing $n$ discrete flaws not to fail under a given far-field stress of $\sigma$ is (Chawla and Meyers, 1999):

$$
\begin{aligned}
P_{V 0}\left(\sigma ; \sigma_{0}, m\right) & =\operatorname{Pr}\left\{\sigma_{\min }>\sigma\right\}=1-\operatorname{Pr}\left\{\sigma_{\min } \leq \sigma\right\} \\
& =\exp \left[-\left(\frac{\sigma}{\sigma_{0}}\right)^{m}\right]
\end{aligned}
$$

where Pr stands for the probability for the condition in the curly bracket to be true, $\sigma_{\min }$ is the minimum value out of all $n$ flaw strengths $\sigma_{a}$, each of which is affiliated with the individual flaw present in the sub-sample of $V_{0}, m$ is a positive-valued parameter called shape parameter or Weibull modulus and $\sigma_{0}$ is a constant called scale parameter. Due to the one-to-one correspondence between the strength and flaw size given in Equation (1), the cumulative distribution function (CDF) in Equation (2) can now be reformulated into a form having the maximum flaw size $a_{\max }$ as the independent variable:

$$
F_{V 0}\left(a ; a_{0}, m\right)=\operatorname{Pr}\left\{a_{\max } \leq a\right\}=\exp \left[-\left(\frac{a}{a_{0}}\right)^{-\frac{m}{2}}\right]
$$

where $a_{0}$ is the scale parameter for the flaw size distribution. Equation (3) gives the probability of the largest flaw in $V_{-0}$ to be smaller than a certain value $a$. As illustrated in Figure 2A, the entire specimen with the finite volume $V$ can now be thought of as the juxtaposition of $N\left(=V / V_{0}\right)$ such sub-samples, all statistically equivalent, and its statistical characteristics can easily be built from that of the sub-sample using the max-stable nature of the extreme value distribution (Coles et al., 2001). Namely, in order for the whole specimen to survive under a given far-field stress, all of the sub-samples should remain intact simultaneously, and therefore the probability for it to occur becomes (Chawla and Meyers, 1999):

$$
\begin{aligned}
P_{V}\left(\sigma ; \sigma_{0}, m\right) & =P_{V 0}\left(\sigma ; \sigma_{0}, m\right)^{N}=\left\{\exp \left[-\left(\frac{\sigma}{\sigma_{0}}\right)^{m}\right]\right\}^{\frac{V}{V_{0}}} \\
& =\exp \left[-\frac{V}{V_{0}}\left(\frac{\sigma}{\sigma_{0}}\right)^{m}\right]
\end{aligned}
$$

The condition for two different specimens to have the same survival probability leads to the well-known Weibull scaling law:

$$
\frac{\sigma_{1}}{\sigma_{2}}=\left(\frac{V_{2}}{V_{1}}\right)^{\frac{1}{m}}
$$

where $\sigma_{1}$ and $\sigma_{2}$ are the strengths of each sample with volumes $V_{1}$ and $V_{2}$, respectively.

\section{CONFINEMENT EFFECTS ON EXTREME VALUE DISTRIBUTIONS IN BRITTLE NANO-SAMPLES}

From the perspective of weakest link theory, the conventional Weibull scaling law given in Equation (5) emerges as a consequence of the fact that a larger specimen contains more flaws than a smaller one and therefore has a higher probability for its weakest flaw, i.e., the largest due to LEFM, to be weaker than the one in the smaller. Here, one important condition to obtain such scaling relationship is the existence of the common reference sub-sample of $V_{0}$ ensuring the statistical equivalence across different specimens in a variety of external dimensions, because Weibull equation is built upon the statistical distribution formulated as the $N$-power of the CDF of a single sub-sample as in Equation (4). Some requirements to keep such condition satisfactory may include (i) materials synthesis in a mutually comparable way between the different specimens so as for the fabrication processes not to affect the fundamental statistics and (ii) more importantly, sufficiently small flaws compared with the whole specimen sizes so as to warrant the presence of the statistically-uniform sub-samples not influenced by the external dimensions of materials. However, the latter condition gets gradually intractable if the size of a specimen decreases close to typical flaw sizes, as is the case for nanomaterials. In that case, the universal reference sub-sample applicable to all specimens regardless of their external dimensions becomes hard to define. Instead, the coupling of fundamental statistics and the characteristic length, e.g., the thickness of nano-plates or diameter of nano-wires as in Figures 2B,C, needs to be taken into account, further requiring the modification of parameters for flaw size distributions, such as the one presented in Equation (3).

The correlation between the flaw size distribution and characteristic length could be inferred by looking into two different statistical representations formulated with different random variables, i.e., one with the actual and the other with maximum flaw size in a sample. When sufficiently many flaws exist in a material, the probability for a flaw in a sub-sample of $V_{0}$ to have a certain size is usually given by a continuous probability density function (PDF), e.g., the Gaussian distribution. On the 
A

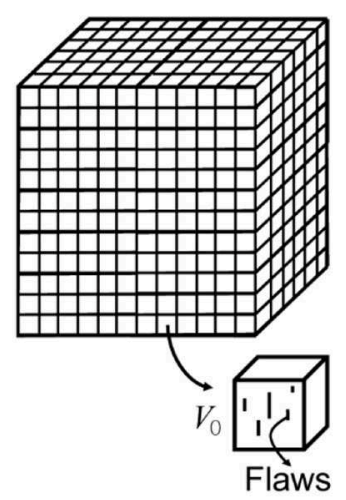

B
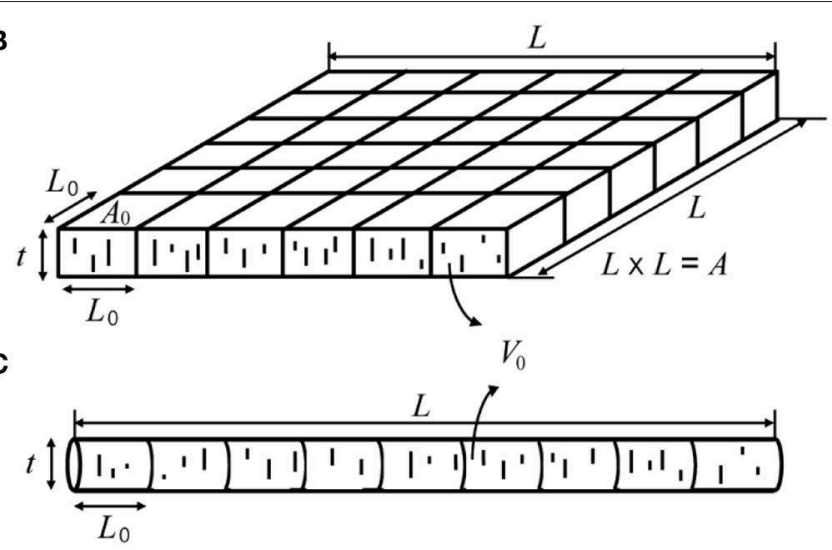

FIGURE 2 | Schematic illustration for sub-sample division of (A) conventional bulk sample, (B) nano-plate, and (C) nano-wire into $V_{0}$.

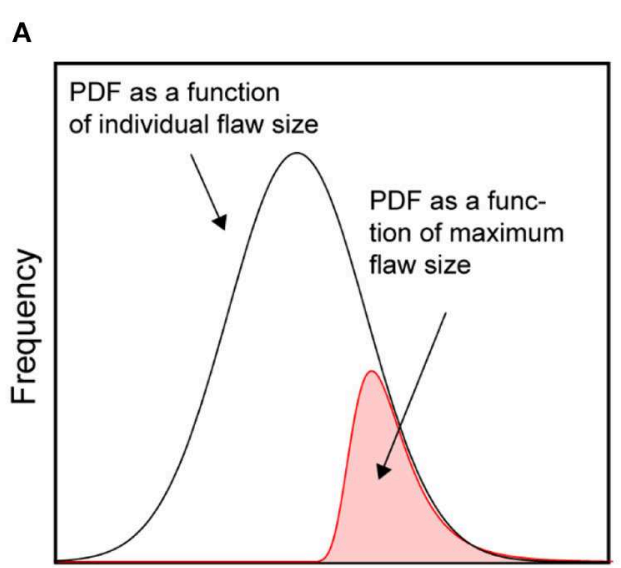

Flaw Size

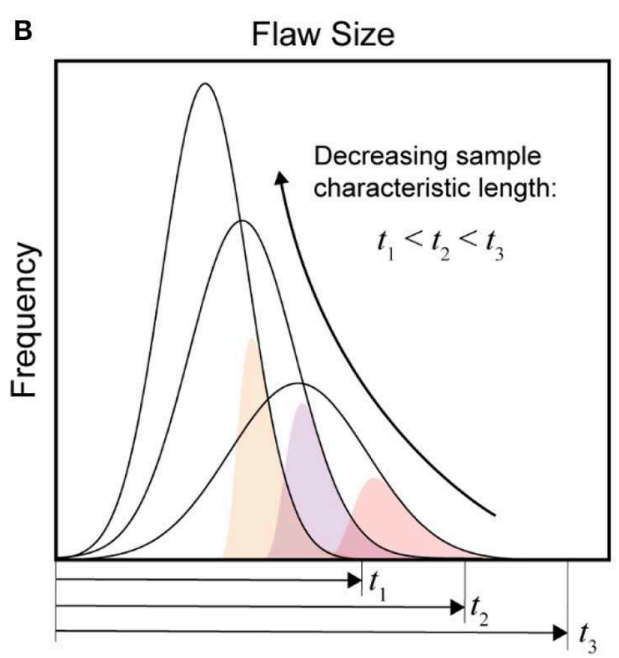

FIGURE 3 | (A) Probability density distribution as a function of the maximum flaw size and its association with that of individual flaw size. (B) Schematic illustration showing the sample size confinement effects on the parent and maximum flaw size distributions.

other hand, when expressed in terms of the size of the largest flaw determined in the sub-sample of $V_{0}$ i.e., by the extreme value formulation, the statistical distribution follows the largeend tail of the parent distribution as shown in Figure 3A (Kotz and Nadarajah, 2000). As long as the specimen is much larger than the width of the parent distribution, the statistical nature of the flaw size remains independent of the external dimensions of the material and the conventional Weibull scaling law still works satisfactorily. However, provided that any flaw can never be larger than the whole body itself, the characteristic length of the sample must bound the width of the parent distribution as it becomes comparable to the flaw sizes (Figure 3B), resulting in a mutual correlation of the former with the latter. Consequently, the extreme value distribution for the maximum flaw sizes should also be coupled with the characteristic length of the specimen, as schematically illustrated in Figure 3B. In this study, in order to incorporate this coupling effect into a statistical formulation, we assume that the scale factor $a_{0}$ in Equation (3) is linearly proportional to the characteristic length $t$, i.e., $a_{0}=\alpha t$, where $\alpha$ is the proportionality constant. Replacing the scale parameter $a_{0}$ by the length-dependent term $\alpha t$ in Equation (3) and taking the derivative, the probability density function prescribing the largest flaw in the whole sample $f_{\mathrm{v}}(a, t ; m)$ becomes:

$$
\begin{aligned}
f_{V}(a, t ; m) & =\frac{d}{d a}\left\{\exp \left[-\frac{V}{V_{0}}\left(\frac{a}{\alpha t}\right)^{-\frac{m}{2}}\right]\right\} \\
& =\frac{m}{2 \alpha t}\left(\frac{V}{V_{0}}\right)\left(\frac{a}{\alpha t}\right)^{-\left(\frac{m}{2}+1\right)} \exp \left[-\frac{V}{V_{0}}\left(\frac{a}{\alpha t}\right)^{-\frac{m}{2}}\right]
\end{aligned}
$$

The expectation value for the largest flaw size in $V$, $\bar{a}$, can be derived as follows:

$$
\bar{a}=\int_{0}^{\infty} a f_{V}(a, t ; m) d a=\alpha t\left(\frac{V}{V_{0}}\right)^{\frac{2}{m}} \Gamma\left(1-\frac{2}{m}\right)
$$

where $\Gamma$ is the gamma function. Furthermore, plugging the maximum flaw size obtained in Equation (7) into the LEFM 


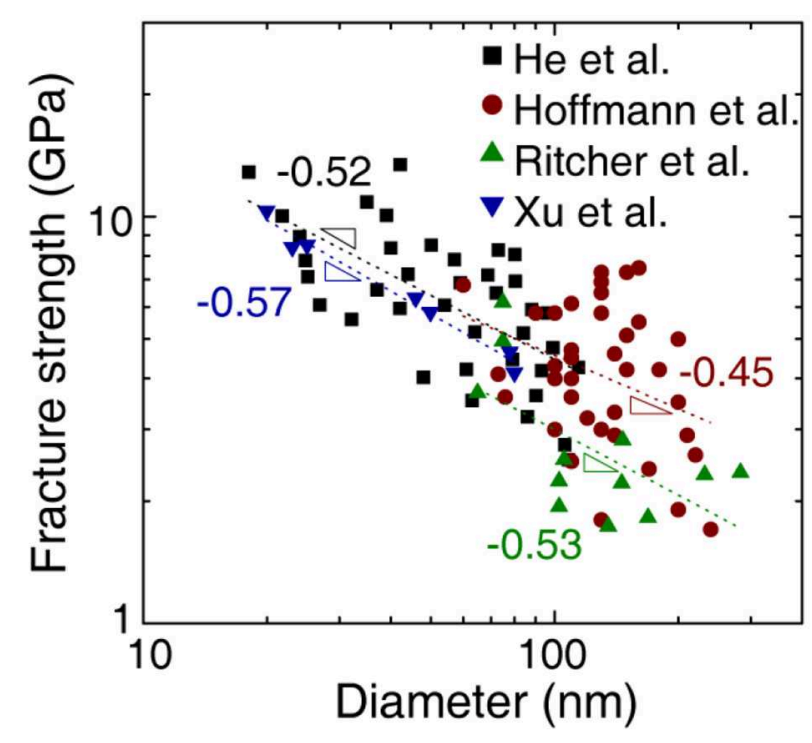

FIGURE 4 | Fracture strength vs. diameter plots from tensile experiments on the brittle ZnO (Hoffmann et al., 2007; Xu et al., 2010; He and Zhu, 2011) and Cu (Richter et al., 2009) nanowires.

strength equation given in Equation (1), the expected value for the strength $\bar{\sigma}$ of a brittle nanomaterial with volume $V$ is:

$$
\bar{\sigma}=\frac{K_{I C}}{\sqrt{\pi \bar{a}}} F(\bar{\varphi})=\frac{G(\beta, v) F(\bar{\varphi})}{\sqrt{\alpha \Gamma\left(1-\frac{2}{m}\right)}} \frac{K_{I C}}{\sqrt{\pi t}}\left(\frac{V_{0}}{V}\right)^{\frac{1}{m}}
$$

where $\bar{\varphi}=\frac{\bar{a}}{t}=\alpha\left(V / V_{0}\right)^{\frac{2}{m}} \Gamma(1-2 / m)$, and $G(\beta, \nu)$ is the geometric factor expressed as a function of the Poisson's ratio $v$, and the inclination angle of the flaw with respect to the loading axis $\beta$ which varies between 0 and $\pi / 2$. When an isotropicallydistributed flaw orientation is assumed, $G(\beta, \nu)$ becomes $2 / \pi$ with $v \sim 0.25$ (Jayatilaka and Trustrum, 1977; Trustrum and Jayatilaka, 1983). Here, it is noteworthy that the inverse-squareroot dependence of the fracture strength on the characteristic length of the specimen, $t$, newly appears as the result of confinement effect on the flaw size distribution in addition to the conventional Weibull scaling term $\left(V_{0} / V\right)^{1 / m}$. The approach presented in this study is applicable to any homogeneous brittle nanomaterial whose failure is governed by the LEFM-based Griffith fracture criterion (Griffith, 1921). The exact length scale below which the confinement effect on the Weibull distribution becomes non-negligible will vary, depending on the type of material and quality of the synthesis process. In the following section, we will confirm the good agreement of our model with experimental data collected from samples under a few 100 nanometers (Hoffmann et al., 2007; Richter et al., 2009; Xu et al., 2010; He and Zhu, 2011).

\section{CASE OF 1D AND 2D NANOMATERIALS}

The equation for the fracture strength shown in Equation (8) can be further developed for the $1 \mathrm{D}$ and $2 \mathrm{D}$ nanomaterials having simple geometry, such as nano-wires or nano-plates. With the help of illustrations given in Figures 2B,C the $V_{0} / V$ term that appears in Equation (8) can be simplified into $L_{0} / L$ and $A_{0} / A$ for nano-wires and nano-plates, respectively, where $L$ and $L_{0}$ are the lengths of the whole and a sub-sample of the nano-wires, $A$ and $A_{0}$ are the areas of the whole and a sub-sample of the nano-plates, respectively. Then, according to Equation (8) the mean strengths for each type of material under uniaxial tensile loading becomes:

$$
\left\{\begin{array}{l}
\bar{\sigma}_{1 D}(t, L ; m)=\frac{G(\beta, \nu) F(\bar{\varphi}(L ; m))}{\sqrt{\alpha \Gamma\left(1-\frac{2}{m}\right)}} \frac{K_{I C}}{\sqrt{\pi t}}\left(\frac{L_{0}}{L}\right)^{\frac{1}{m}}, \\
\bar{\sigma}_{2 D}(t, A ; m)=\frac{G(\beta, v) F(\bar{\varphi}(A ; m))}{\sqrt{\alpha \Gamma\left(1-\frac{2}{m}\right)}} \frac{K_{I C}}{\sqrt{\pi t}}\left(\frac{A_{0}}{A}\right)^{\frac{1}{m}} .
\end{array}\right.
$$

Furthermore, if we restrict our interest to the set of samples whose axial lengths ( $L$ for nano-wires) or areas ( $A$ for nanoplates) remain constant, but only the characteristic lengths $(t)$, i.e., the diameter (nano-wires) or the thickness (nano-plates), vary, the mean strength depends on $t$, following the reciprocal square-root relation, i.e., $\sigma \infty t^{-1 / 2}$. This result is, in principle, equivalent with the scaling law offered by Gao et al. for brittle nano-plates (Gao et al., 2003). The equations derived in this work are applicable only to the nanomaterials satisfying the following conditions: (i) failure of materials in different sizes should predominantly be governed by the same type of flaw. This condition may require the specimens to be prepared by the same fabrication procedure. (ii) The volume of the specimens should be large enough to include sufficiently many sub-samples to ensure the statistical consistency. Simultaneously, the critical dimension of the sample should be small enough, approximately a few tens to a few 100 nanometers, so as for the volumetric confinement effect to emerge. The uniaxial fracture strengths from a set of brittle nanowires or nanoplates differing in their sizes but with identical microstructural features would be an ideal example with which to verify the model presented in this work. To the best of our knowledge, there exist only a limited number of experimental data available until now. Nonetheless, in Figure 4, we present some examples of actual experimental data collected from the literature that reports the uniaxial tensile strengths of brittle nano-whiskers made of $\mathrm{ZnO}$ (Hoffmann et al., 2007; Xu et al., 2010; He and Zhu, 2011) and single crystalline $\mathrm{Cu}$ (Richter et al., 2009).Two important features are noteworthy here: (i) clear demonstration of the reciprocal square-root relation between the diameter $t$ and fracture strength and (ii) large scatter in the data. The former serves as the strong evidence for our work, and the latter indicates that the statistical fluctuation originated from the large Weibull modulus is still dominant in this regime.

\section{CONCLUSIONS}

In conclusion, we suggest a new theoretical framework for fracture strength of brittle nanomaterials combining 
the confinement effect on maximum flaw size distribution into the conventional Weibull statistics. By modifying the scale factor of the maximum crack size distribution function to be proportional to the characteristic length of the specimen, we successfully derive the integrated formula of fracture strength for nanomaterials which contains both the conventional Weibull and newly-added confinement terms in its scaling relationship. We further verified the validity of our equation in the range where the characteristic length is smaller than $300 \mathrm{~nm}$ by fitting it to actual experimental data collected for brittle nanowhiskers. This theoretical approach offers a foundation for the design of strain engineering and enables brittle nanomaterials to be more reliable and stable for their practical applications.

\section{REFERENCES}

Anderson, T. L. (2017). Fracture Mechanics: Fundamentals and Applications. New York, NY: CRC Press.

Carpinteri, A. (1994). Scaling laws and renormalization groups for strength and toughness of disordered materials. Int. J. Solids Struct. 31, 291-302. doi: 10.1016/0020-7683(94)90107-4

Carpinteri, A., and Pugno, N. M. (2004). Scale-effects on mean and standard deviation of the mechanical properties of condensed matter: an energy-based unified approach. Int. J. Fract. 128, 253-261. doi: 10.1023/B:FRAC.0000040988.61253.05

Carpinteri, A., and Pugno, N. M. (2005). Are scaling laws on strength of solids related to mechanics or to geometry. Nat. Mater. 4, 421-423. doi: $10.1038 /$ nmat 1408

Chawla, K. K., and Meyers, M. (1999). Mechanical Behavior of Materials. New York, NY: Prentice Hall Upper Saddle River.

Coles, S., Bawa, J., Trenner, L., and Dorazio, P. (2001). An Introduction to Statistical Modeling of Extreme Values, Vol. 208. London: Springer.

Gao, H., Ji, B., Jäger, I. L., Arzt, E., and Fratzl, P. (2003). Materials become insensitive to flaws at nanoscale: lessons from nature. Proc. Natl. Acad. Sci. U.S.A. 100, 5597-5600. doi: 10.1073/pnas.0631609100

Gilman, J. J. (2003). Electronic Basis of the Strength of Materials. New York, NY: Cambridge University Press.

Griffith, A. A. (1921). The phenomena of rupture and flow in solids. Phil. Trans. $R$. Soc. Lond. A 221, 163-198. doi: 10.1098/rsta.1921.0006

He, M.-R., and Zhu, J. (2011). Defect-dominated diameter dependence of fracture strength in single-crystalline $\mathrm{ZnO}$ nanowires: in situ experiments. Phy. Rev. B. 83:161302. doi: 10.1103/PhysRevB.83.161302

Hoffmann, S., Östlund, F., Michler, J., Fan, H. J., Zacharias, M., Christiansen, S. H., and Ballif, C. (2007). Fracture strength and Young's modulus of $\mathrm{ZnO}$ nanowires. Nanotechnology. 18, 1-6. doi: 10.1088/0957-4484/18/20/205503

Jang, D., and Greer, J. R. (2010). Transition from a strong-yet-brittle to a strongerand-ductile state by size reduction of metallic glasses. Nat. Mater. 9, 215-219. doi: $10.1038 /$ nmat 2622

Jang, D., Meza, L. R., Greer, F., and Greer, J. R. (2013). Fabrication and deformation of three-dimensional hollow ceramic nanostructures. Nat. Mater. 12, 893-898. doi: $10.1038 /$ nmat 3738

Jayatilaka, A. D. S., and Trustrum, K. (1977). Statistical approach to brittle fracture. J. Mater. Sci. 12, 1426-1430. doi: 10.1007/BF00540858

Kittel, C., and McEuen, P. (1996). Introduction to Solid State Physics, Vol. 8. New York, NY: Wiley.

Kotz, S., and Nadarajah, S. (2000). Extreme Value Distributions: Theory and Applications. London: World Scientific.

Le, J. L., and BaŽant, Z. P. (2012). Scaling of static fracture of quasi-brittle structures: strength, lifetime, and fracture kinetics. J. Appl. Mech. 79:031006. doi: 10.1115/1.4005881

Li, J., Shan, Z., and Ma, E. (2014). Elastic strain engineering for unprecedented materials properties. MRS Bull. 39, 108-114. doi: 10.1557/mrs.2014.3

\section{DATA AVAILABILITY STATEMENT}

Publicly available datasets were analyzed in this study. This data can be found here: doi: 10.1007/s12274-010-1030-4, doi: 10.1103/PhysRevB.83.161302, doi: 10.1021/n19015107, doi: 10.1088/0957-4484/18/20/205503.

\section{AUTHOR CONTRIBUTIONS}

All authors conceived the research, formulated the theory, discussed the results, and wrote the manuscript.

\section{FUNDING}

The authors acknowledge financial support from National Research Foundation of Korea (NRF-2019M2D2A1A02038972).

Pang, S. D., BaŽant, Z. P., and Le, J. L. (2008). Statistics of strength of ceramics: finite weakest-link model and necessity of zero threshold. Int. J. Fract. 154, 131-145. doi: 10.1007/s10704-009-9317-8

Pelleg, J. (2014). Mechanical Properties of Ceramics, Vol. 213. Cham: Springer Science \& Business.

Pugno, N. M., and Ruoff, R. S. (2004). Quantized fracture mechanics. Philosoph. Mag. 84, 2829-2845. doi: 10.1080/14786430412331280382

Pugno, N. M., and Ruoff, R. S. (2006). Nanoscale Weibull statistics. J. Appl. Phys. 99:024301. doi: 10.1063/1.2158491

Quinn, J. B., and Quinn, G. D. (2010). A practical and systematic review of Weibull statistics for reporting strengths of dental materials. Dent. Mater. 26, 135-147. doi: 10.1016/j.dental.2009.09.006

Richter, G., Hillerich, K., Gianola, D. S., Monig, R., Kraft, O., and Volkert, C. A. (2009). Ultrahigh strength single crystalline nanowhiskers grown by physical vapor deposition. Nano Lett. 9, 3048-3052. doi: 10.1021/nl9015107

Sun, G., Pang, J, H., L., Zhou, J., Zhang, Y., Zhan, Z., and Zheng, L. (2012). A modified Weibull model for tensile strength distribution of carbon nanotube fibers with strain rate and size effects. Appl. Phys. Lett. 101:131905. doi: $10.1063 / 1.4754709$

Suresh, S., and Li, J. (2008). Materials science: deformation of the ultra-strong, Nature 456, 716-717. doi: 10.1038/456716a

Taloni, A., Vodret, M., Costantini, G., and Zapperi, S. (2018). Size effects on the fracture of microscale and nanoscale materials. Nat. Rev. Mater. 3, 211-224. doi: 10.1038/s41578-018-0029-4

Trustrum, K., and Jayatilaka, A. D. S. (1983). Applicability of Weibull analysis for brittle materials. J. Mater. Sci. 18, 2765-2770. doi: 10.1007/BF005 47593

Wang, J., Sansoz, F., Huang, J., Liu, Y., Sun, S., Zhang, Z., et al. (2013). Near-ideal theoretical strength in gold nanowires containing angstrom scale twins. Nat. Commun. 4:1742. doi: 10.1038/ncomms2768

Weibull, W. (1951). A statistical distribution function of wide applicability. J. Appl. Mech. 103, 293-297.

Xu, F., Qin, Q., Mishra, A., Gu, Y., and Zhu, Y. (2010). Mechanical properties of $\mathrm{ZnO}$ nanowires under different loading modes. Nano Res. 3, 271-280. doi: 10.1007/s12274-010-1030-4

Zhu, T., and Li, J. (2010). Ultra-strength materials. Prog. Mater. Sci. 55, 710-757. doi: 10.1016/j.pmatsci.2010.04.001

Conflict of Interest: The authors declare that the research was conducted in the absence of any commercial or financial relationships that could be construed as a potential conflict of interest.

Copyright $\odot 2019$ Shin and Jang. This is an open-access article distributed under the terms of the Creative Commons Attribution License (CC BY). The use, distribution or reproduction in other forums is permitted, provided the original author $(s)$ and the copyright owner(s) are credited and that the original publication in this journal is cited, in accordance with accepted academic practice. No use, distribution or reproduction is permitted which does not comply with these terms. 